Western University

Scholarship@Western

Mechanical and Materials Engineering

Mechanical and Materials Engineering

Publications

Department

2020

\title{
Exact solutions to the three-dimensional Navier-Stokes equations using the extended Beltrami method
}

Nolan J. Dyck

Western University, ndyck@uwo.ca

Anthony G. Straatman

uwo, agstraat@uwo.ca

Follow this and additional works at: https://ir.lib.uwo.ca/mechanicalpub

Part of the Materials Science and Engineering Commons, and the Mechanical Engineering Commons

\section{Citation of this paper:}

Dyck, Nolan J. and Straatman, Anthony G., "Exact solutions to the three-dimensional Navier-Stokes equations using the extended Beltrami method" (2020). Mechanical and Materials Engineering

Publications. 4.

https://ir.lib.uwo.ca/mechanicalpub/4 


\author{
Nolan J. Dyck \\ Department of Mechanical and Materials \\ Engineering, \\ Western University, \\ London, ON, N6A 5B9, Canada \\ e-mail: ndyck@uwo.ca \\ Anthony G. Straatman \\ Department of Mechanical and Materials \\ Engineering, \\ Western University, \\ London, ON, N6A 5B9, Canada \\ e-mail: astraatman@eng.uwo.ca
}

\section{Exact Solutions to the Three- Dimensional Navier-Stokes Equations Using the Extended Beltrami Method}

In a 1966 publication, Chi-Yi Wang used the streamfunction in concert with the vorticity equations to develop a methodology for obtaining exact solutions to the incompressible Navier-Stokes equations, now known as the extended Beltrami method. In Wang's approach, the vorticity is represented by the sum of a linear function of the streamfunction and an assumed auxiliary function, such that the vorticity equation can be reduced to a quasi-linear partial differential equation, and exact solutions are obtainable for many choices of the auxiliary function. In the present work, a natural extension of Wang's formulation to three-dimensional flows in arbitrary orthogonal curvilinear coordinates has been derived, wherein two auxiliary functions are formed at the outset, with the caveat that the pressure and velocity components may vary in two spatial dimensions. As is the case with two-dimensional extended Beltrami flows, exact solutions are only obtainable when the forms of the auxiliary functions are "simple enough" to render the governing equations solvable. To demonstrate the solutions which may be obtained using the extended formulation, the well-known Kovasznay flow is generalized to a three-dimensional flow. A unique solution in plane polar coordinates is found. An extension to the solution to Burgers vortex has been derived and discussed in the context of existing literature. Finally, a new $3 D$ swirling flow solution which is the angular analogue to Kovasznay flow has been developed.

[DOI: 10.1115/1.4044927]

Keywords: aerodynamics, internal flow

\section{Introduction}

The Navier-Stokes equations describe mathematically the motions of a fluid where pressure forces and viscous forces are significant. When the flow may be considered incompressible and isothermal, the governing equations may be written in dimensionless form:

$$
\nabla \cdot \mathbf{u}=0
$$

$$
\frac{\partial \mathbf{u}}{\partial t}+(\mathbf{u} \cdot \nabla) \mathbf{u}=-\nabla p+\frac{1}{\operatorname{Re}} \nabla^{2} \mathbf{u}
$$

where $\mathbf{u}$ is the velocity, $t$ is the time, $\nabla$ is the gradient operator, $p$ is the thermodynamic pressure, and the Reynolds number is defined as $\operatorname{Re}=u_{c} x_{c} / \nu$. The parameters $u_{c}$ and $x_{c}$ are characteristic velocity and length scales, respectively, and $\nu$ is the kinematic viscosity. Equation (2) introduces significant complexity in solving fluid flow problems, as the second term (known as the advection term) renders the equation non-linear, while the fourth term (representing the contribution of viscous forces) contains derivatives of the second order. No general solution to the systems 1-2 has been found, nor is there a single solution technique which can be used to obtain all the solutions found in the literature. Many fluid flow problems encountered in engineering contain complicated geometries and boundary conditions, which can be intractable without the aid of numerical methods. Nevertheless, exact solutions, wherever they can be obtained, can give important insights into the relationships between solution parameters, provide closed-form expressions of

Contributed by the Applied Mechanics Division of ASME for publication in the Journal of APPLIEd Mechanics. Manuscript received June 11, 2019; final manuscript received September 17, 2019; published online September 19, 2019. Assoc. Editor: N. R. Aluru. base flows as starting points for linear and non-linear stability analyses, and serve as benchmark solutions for testing computer codes. Fluid dynamics textbooks such as Ref. [1] contain many elementary solutions to the Navier-Stokes equations but are not exhaustive. Readers are directed to the reviews of Wang [2,3] and the book by Drazin and Riley [4] for a comprehensive summary of the exact solutions found in the literature.

An exact solution to the Navier-Stokes equations is a solution

(1) which may be expressed in terms of elementary functions

(2) expressible in terms of one or more ordinary differential equations

(3) that may be expressed as a combination of items 1 and 2

Exact solutions are most commonly obtained by one of the three general approaches: analysis of unidirectional or essentially unidirectional flows, similarity solutions, and Beltrami and Beltrami-related flows. Since the approach described in the present work extends a Beltrami-related solution technique, only similar solution techniques will be reviewed here.

Taking the curl of Eq. (2) gives the vorticity equation

$$
\frac{\partial \omega}{\partial t}+\nabla \times(\omega \times \mathbf{u})=\frac{1}{\operatorname{Re}} \nabla^{2} \omega
$$

where $\omega=\nabla \times u$ is the vorticity. Flows in which the vorticity is 0 everywhere are called irrotational [1]. If the flow is assumed to be planar and cross-flow is absent, two of the vorticity components are zero and only one equation survives. Several researchers have addressed this general case by guessing various functional forms of the streamfunction and solving the vorticity equation, including Polyanin and Aristov [5], Polyanin and Zhurov [6], and Kumar and Kumar [7]. These solutions often include complicated unsteady terms, which are difficult to visualize. 
1.1 Beltrami Flows. In a Beltrami flow, the vorticity is parallel to the streamlines everywhere and the cross product $\omega \times \mathbf{u}$ vanishes. This condition is primarily useful for solving flow problems with negligible viscous forces (i.e., $\nu \rightarrow 0$ ), where the flow is still rotational. In this case, it can be shown (see Sec. 3.6 of Ref. [1]) that the Bernoulli function remains constant throughout the flow domain.

1.2 Generalized Beltrami Flows. When the alternative condition $\nabla \times(\omega \times \mathbf{u})=0$ holds, the flow is called a generalized Beltrami flow. Most often, this approach has been adopted to study 2D planar and $2 \mathrm{D}$ axisymmetric flows without cross-flow or swirl, respectively. In these cases, two of the vorticity components vanish, and the solution is constrained by the definition of the third component of vorticity, the generalized Beltrami flow condition, and the remaining terms in Eq. (3). For the special case when the vorticity is constant, the three equations reduce to a single Poisson-type equation in terms of the streamfunction. Working in Cartesian coordinates, Tsien [8] developed two solutions describing the influence of a line source and a line vortex on a shear flow, respectively. Wang [9] presented a solution representing "shear flow over convection cells" and another solution [3] describing the oblique impingement of two jets. In cylindrical coordinates, a similar simplification yields a Poisson-like partial differential equation in terms of the streamfunction. Specific solutions include Hill's spherical vortex [10] and augmentations thereof: O'Brien's ellipsoidal vortex [11], Wang's toroidal vortex [9], and the solutions given by Fujimoto et al. [12]. Recently, Joseph [13] has shown that there is a general family of solutions in terms of generalized hypergeometric series which contain, in addition to Hill's, Wang's, and Fujimoto et al.'s solutions as special cases, "Fig. 8" and butterfly vortices for different choices of the coefficients. Berker [14] gives a homogeneous solution in terms of Bessel functions and exponential functions. Terrill [15], apparently independently, developed a solution describing the axisymmetric motion of a fluid in a porous pipe which is an expression of Berker's solution.

Saccomandi studied steady and unsteady pseudo-plane flows wherein only the third component of the advection term is required to vanish $[16,17]$ in a Cartesian coordinate system (i.e., $\nabla \times(\omega \times \mathbf{u}) \cdot \hat{\mathbf{k}}=0$, where $\hat{\mathbf{k}}$ is the unit vector pointing in the $z$-direction). In these works, pseudo-plane flows are those in which the motion is restricted to the $x-y$ plane, but the streamfunction retains dependence on the $z$ coordinate.

Weinbaum and O'Brien [18] have collected and expanded the set of known solutions to the Navier-Stokes equations under the generalized Beltrami condition where out-of-plane velocity components (called cross-flow for Cartesian systems and swirl in axisymmetric cylindrical coordinate systems) are significant, with the stipulation that the streamfunction and out-of-plane velocity components vary only with in-plane coordinates and time.

1.3 Extended Beltrami Flows. Wang [19] presented another solution technique, wherein the third component of vorticity is expressed as a function of the streamfunction plus an auxiliary function of the assumed form. The rationale for this choice as well as existing solutions obtainable using this method will be discussed in Sec. 3.

Our initial motivation for undertaking the present work was to develop an exact solution that resembles Ranque-Hilsch flow; however, we quickly realized that our derivation (described in Sec. 6.2) was based on a more general extension of the extended Beltrami method, which has not yet been detailed in the literature. In the present work, we aim to systematically reduce the governing equations according to the assumptions of the extended Beltrami method, while keeping the formulation coordinate system independent.

In Sec. 2, we develop the streamfunction-vorticity equation set in general orthogonal curvilinear coordinates, laying the groundwork for Sec. 3 where the extended Beltrami method surmised by Wang is extended to three-dimensional flows. In Sec. 4, we show that our formulation reduces to that of Wang for 2D planar flows and revisit some familiar solutions. In Sec. 5, we briefly demonstrate that all planar solutions of Sec. 4 support a non-uniform out-of-plane velocity distribution, which is easily obtainable from the planar solution. In Sec. 6, we study axisymmetric flows with swirl; we revisit Burgers vortex and related flows, and we develop a new solution, which can be considered the angular analogue of Kovasznay flow. We summarize our contributions in Sec. 7.

\section{Governing Equations}

In this section, the governing equations (2) and (3) will be further developed in preparation for deriving the working equations in Sec. 3. We first write the continuity equation (1) in terms of a general orthogonal curvilinear coordinate system $\left(x_{1}, x_{2}, x_{3}\right)$, wherein velocity and pressure are independent of the third coordinate, $x_{3}$ :

$$
\frac{\partial u_{1}}{\partial x_{1}}+\frac{\partial u_{2}}{\partial x_{2}}=0
$$

Furthermore, the velocity can be represented in terms of a pair of streamfunctions, $\psi$ and $\gamma[1]$ :

$$
\mathbf{u}=\nabla \times(\psi \nabla \gamma)+u_{3} \hat{\mathbf{e}}_{3}
$$

where the first term on the right-hand side of Eq. (5) is a vector lying in the $x_{1}-x_{2}$ surface and the final term is a vector pointing in the $x_{3}$ direction. Inserting Eq. (5) into Eq. (4), we find $\gamma=x_{3}$, and the velocity may be expressed as

$$
\mathbf{u}=\frac{1}{h_{3}}(\nabla \psi) \times \hat{\mathbf{e}}_{3}+u_{3} \hat{\mathbf{e}}_{3}=\frac{1}{h_{2} h_{3}} \frac{\partial \psi}{\partial x_{2}} \hat{\mathbf{e}}_{1}-\frac{1}{h_{1} h_{3}} \frac{\partial \psi}{\partial x_{1}} \hat{\mathbf{e}}_{2}+u_{3} \hat{\mathbf{e}}_{3}
$$

where $h_{i}=h_{i}\left(x_{1}, x_{2}\right)$ and $\mathbf{e}_{i}, i \in\{1,2,3\}$, are the corresponding scale factors and unit vectors, respectively. Note that the present analysis excludes coordinate systems in which any of the scale factors vary with the third coordinate, $x_{3}$, such as conical, confocal ellipsoidal, and confocal paraboloidal coordinate systems. The vorticity may also be expressed in terms of the streamfunction:

$$
\begin{aligned}
\omega=\nabla \times \mathbf{u}= & \frac{1}{h_{2} h_{3}} \frac{\partial\left(h_{3} u_{3}\right)}{\partial x_{2}} \hat{\mathbf{e}}_{1}-\frac{1}{h_{1} h_{3}} \frac{\partial\left(h_{3} u_{3}\right)}{\partial x_{1}} \hat{\mathbf{e}}_{2} \\
& -\frac{1}{h_{1} h_{2}}\left[\frac{\partial}{\partial x_{1}}\left(\frac{h_{2}}{h_{1} h_{3}} \frac{\partial \psi}{\partial x_{1}}\right)+\frac{\partial}{\partial x_{2}}\left(\frac{h_{1}}{h_{2} h_{3}} \frac{\partial \psi}{\partial x_{2}}\right)\right] \hat{\mathbf{e}}_{3}
\end{aligned}
$$

As it will be seen later, it is useful to define the pseudo-vorticity, a modified definition of the third component of the vorticity vector [19]:

$$
\xi=\frac{\omega_{3}}{h_{3}}=\frac{-1}{h_{1} h_{2} h_{3}}\left[\frac{\partial}{\partial x_{1}}\left(\frac{h_{2}}{h_{1} h_{3}} \frac{\partial \psi}{\partial x_{1}}\right)+\frac{\partial}{\partial x_{2}}\left(\frac{h_{1}}{h_{2} h_{3}} \frac{\partial \psi}{\partial x_{2}}\right)\right]=-\frac{D^{2}(\psi)}{V}
$$

where $V=h_{1} h_{2} h_{3}$. The cross-flow velocity may be written as $\sigma=$ $h_{3} u_{3}$. Using these definitions, the $u_{3}$-momentum and $\omega_{3}$-vorticity equations may be expressed as

$$
\begin{gathered}
V \frac{\partial \sigma}{\partial t}+K(\sigma, \psi)=\frac{h_{3}^{2} D^{2}(\sigma)}{\operatorname{Re}} \\
V \frac{\partial \xi}{\partial t}+K(\xi, \psi)-K\left(\frac{\sigma}{h_{3}^{2}}, \sigma\right)=\frac{D^{2}\left(h_{3}^{2} \xi\right)}{\operatorname{Re}}
\end{gathered}
$$

where $K(a, b)=\left(\partial a / \partial x_{1}\right)\left(\partial b / \partial x_{2}\right)-\left(\partial b / \partial x_{1}\right)\left(\partial a / \partial x_{2}\right)$ is the Jacobian. Equations (9) and (10) have been derived in the Appendix. While the pressure $p$ may be determined through the first two components of Eq. (2), the first two equations in (3) are superfluous in the present formulation [20]. Thus, solutions to the system of Eqs. (8)-(10) are also solutions to the Navier-Stokes equations. These equations are the point of departure for Polyanin [21] and Polyanin and Zhurov [6], who have assumed various forms of the streamfunction $\psi$ in 

for 2D planar flows. Lewellen [22] has developed the same equations for the case of axisymmetric cylindrical coordinates and derived an asymptotic series solution to describe the vortex motion wherein a specific dimensionless parameter is considered small. Granger [23] has adopted Lewellen's formulation and derived approximate solutions using a power series formulation.

\section{Wang's Approach}

Wang [2,19] surmised the solution techniques of earlier researchers including Kovasznay [24], Taylor [25], Kelvin, and others, suggesting that, in the absence of flow normal to the $\psi$-plane, the pseudo-vorticity of any axisymmetric or $2 \mathrm{D}$ flow could be represented as

$$
\xi=g(\psi)+\chi\left(x_{1}, x_{2}, t\right)=-\frac{D^{2}(\psi)}{V}
$$

where $g(\psi)$ and $\chi\left(x_{1}, x_{2}\right)$ are known functions. Wang rationalizes this choice by observing that when Eq. (11) is inserted into Eq. (10) (with $\sigma=0$ ), the advection terms involving $g$ are selfcancelling. Furthermore, under this formulation, the viscous term in Eq. (10) contains $D^{2}\left(h_{3}^{2} \xi\right)$, which in turn may be replaced using Eq. (11) twice. When $g(\psi) \propto \psi$, Eq. (10) reduces to a first-order quasi-linear partial differential equation, which is readily solvable for many choices of $\chi$. The aim of the present work is to develop similar relations to yield solutions for cases where the cross-flow $\sigma$ may vary with $x_{1}, x_{2}$. While Wang's original work developed Eq. (10), including the out-of-plane velocity component, the Navier-Stokes equations were not explicitly considered and only solutions to 2D planar flows were derived. To the best of our knowledge, no exact solutions extending Wang's approach to three dimensions exist in the open literature.

To begin, $\xi$ and $\sigma$ are first expressed in terms of $\psi$ and $t$ such that the advection terms are reduced to 0. Beginning with the advection terms of Eqs. (9) and (10), the reasoning of Leprovost et al. [26] has been followed, while generalizing the derivation for unsteady flows in an arbitrarily orthogonal curvilinear coordinate system:

$$
K(\sigma, \psi)=0
$$

the absence of cross-flow $(\sigma=0)$ and arrived at a variety of solutions

$$
K(\xi, \psi)-K\left(\frac{\sigma}{h_{3}^{2}}, \sigma\right)=0
$$

Equation (12) is satisfied when $\sigma=F(\psi, t)$. Then, using the identity

$$
K\left(\frac{\sigma}{h_{3}^{2}}, F\right)=K\left(\frac{\sigma}{h_{3}^{2}} \frac{\partial F}{\partial \psi}, \psi\right)
$$

Equation (13) may be rewritten as

$$
K\left(\xi-\frac{F}{h_{3}^{2}} \frac{\partial F}{\partial \psi}, \psi\right)=0
$$

which suggests $\xi-\left(F / h_{3}^{2}\right) \partial F / \partial \psi=G(\psi, t)$. The desired forms of the cross-flow velocity and pseudo-vorticity are

$$
\begin{gathered}
\sigma=F(\psi, t) \\
\xi=\frac{F}{h_{3}^{2}} \frac{\partial F}{\partial \psi}+G(\psi, t)
\end{gathered}
$$

Together with Eq. (8), these are the Bragg-Hawethorne or Squire-Long equations presented for a general curvilinear orthogonal coordinate system $[27,28]$. In their present state, application of Eqs. (16) and (17) to the systems 9 and 10 would leave only the unsteady and viscous terms, and generalized Beltrami flows can be obtained by solving the reduced equation set. Instead, we follow Wang's approach [19] and add auxiliary functions to $\sigma$ and $\xi$ :

$$
\begin{gathered}
\sigma=F(\psi, t)+\Phi\left(x_{1}, x_{2}, t\right) \\
\xi=\frac{F}{h_{3}^{2}} \frac{\partial F}{\partial \psi}+G(\psi, t)+X\left(x_{1}, x_{2}, t\right)=-\frac{D^{2}(\psi)}{V}
\end{gathered}
$$

Substituting the above forms into Eqs. (9) and (10) and simplifying yield

$$
V \frac{\partial F}{\partial t}+V \frac{\partial \Phi}{\partial t}+K(\Phi, \psi)=\frac{h_{3}^{2}}{\operatorname{Re}}\left\{\frac{\partial F}{\partial \psi} D^{2}(\psi)+\frac{\partial^{2} F}{\partial \psi^{2}} \bar{\psi}^{2}+D^{2}(\Phi)\right\}
$$

where

$$
\begin{aligned}
\frac{V}{h_{3}^{2}}\left(\frac{\partial F}{\partial t} \frac{\partial F}{\partial \psi}+F \frac{\partial^{2} F}{\partial \psi \partial t}\right)+V \frac{\partial G}{\partial t}+V \frac{\partial X}{\partial t}+K(X, \psi)+\frac{2}{h_{3}^{3}}\left[(F+\Phi) K\left(h_{3}, \Phi\right)+\Phi \frac{\partial F}{\partial \psi} K\left(h_{3}, \psi\right)\right] \\
=\frac{1}{\operatorname{Re}}\left\{\left[\left(\frac{\partial F}{\partial \psi}\right)^{2}+F \frac{\partial^{2} F}{\partial \psi^{2}}+h_{3}^{2} \frac{\partial G}{\partial \psi}\right] D^{2}(\psi)+\frac{\partial}{\partial \psi}\left[\left(\frac{\partial F}{\partial \psi}\right)^{2}+F \frac{\partial^{2} F}{\partial \psi^{2}}+h_{3}^{2} \frac{\partial G}{\partial \psi}\right] \bar{\psi}^{2}\right\} \\
\quad+\frac{1}{\operatorname{Re}}\left\{D^{2}\left(h_{3}^{2}\right) G+D^{2}\left(h_{3}^{2} X\right)+4 \frac{\partial G}{\partial \psi}\left[\frac{h_{2}}{h_{1}} \frac{\partial h_{3}}{\partial x_{1}} \frac{\partial \psi}{\partial x_{1}}+\frac{h_{1}}{h_{2}} \frac{\partial h_{3}}{\partial x_{2}} \frac{\partial \psi}{\partial x_{2}}\right]\right\}
\end{aligned}
$$

Equations (20) and (23) are cumbersome and unlikely to yield solutions while the non-linear $\bar{\psi}^{2}$ terms are present. To eliminate these terms, we set $F, G \propto \psi$. Additionally, from hereon-in we narrow our focus to steady solutions, dropping the dependence on $t$, so Eqs. (18) and (19) are reduced to

$$
\begin{aligned}
& F(\psi)=d_{F} \psi \\
& G(\psi)=d_{G} \psi
\end{aligned}
$$

and the auxiliary functions are relabeled $\Phi\left(x_{1}, x_{2}\right)=\phi\left(x_{1}, x_{2}\right)$ and $X\left(x_{1}, x_{2}\right)=\chi\left(x_{1}, x_{2}\right)$. With these simplifications, the pseudo-vorticity reduces to

$$
\xi=\frac{d_{F}^{2}}{h_{3}^{2}} \psi+d_{G} \psi+\chi=-\frac{D^{2}(\psi)}{V}
$$

and Eqs. (20) and (21) become

$$
K(\phi, \psi)=\frac{1}{\operatorname{Re}}\left\{h_{3}^{2} D^{2}(\phi)-V d_{F}\left(d_{F}^{2} \psi+d_{G} h_{3}^{2} \psi+h_{3}^{2} \chi\right)\right\}
$$




$$
\begin{aligned}
& K(\chi, \psi)+\frac{2}{h_{3}^{3}}\left[\left(d_{F} \psi+\phi\right) K\left(h_{3}, \phi\right)+\phi d_{F} K\left(h_{3}, \psi\right)\right] \\
& =\frac{1}{\operatorname{Re}}\left\{-V\left(d_{F}^{2}+d_{G} h_{3}^{2}\right)\left(\frac{d_{F}^{2}}{h_{3}^{2}} \psi+d_{G} \psi+\chi\right)+d_{G} D^{2}\left(h_{3}^{2}\right) \psi+D^{2}\left(h_{3}^{2} \chi\right)\right. \\
& \left.\quad+4 d_{G}\left[\frac{h_{2}}{h_{1}} \frac{\partial h_{3}}{\partial x_{1}} \frac{\partial \psi}{\partial x_{1}}+\frac{h_{1}}{h_{2}} \frac{\partial h_{3}}{\partial x_{2}} \frac{\partial \psi}{\partial x_{2}}\right]\right\}
\end{aligned}
$$

In principle, Eqs. (25)-(27) may be used to find almost any 3D flow in an orthogonal coordinate system, provided the flow is independent of one of the coordinates and the scale factors of the chosen coordinate system are independent of the same coordinate. In practice, the value of the present formulation is limited by the number of auxiliary functions which are "simple enough" to generate exact solutions. In the following subsections, both existing and new solutions will be found using linear and quadratic auxiliary functions. Further assumptions are required to reach general solutions, and these will be explored in the following sections.

\section{2D Planar Flows}

In the absence of an out-of-plane velocity component (i.e., $\sigma=$ $d_{F}=\phi=0$ ), Eq. (26) is automatically satisfied. In planar coordinate systems, $h_{3}=1$. With these simplifications, Eqs. (27) and (25) reduce to

$$
\begin{gathered}
K(\chi, \psi)=\frac{1}{\operatorname{Re}}\left\{D^{2}(\chi)-d_{G} V\left(d_{G} \psi+\chi\right)\right\} \\
\xi=d_{G} \psi+\chi=-\frac{D^{2}(\psi)}{V}
\end{gathered}
$$

These equations have been solved in the Cartesian coordinate system by many authors to find solutions describing several wellknown flows.

4.1 Cartesian Coordinate System. In Cartesian coordinates $(x, y, z)$, the scale factors become $h_{1}=h_{2}=h_{3}=V=1$. Given the restrictions $\chi=\chi(y)$ and $D^{2}(\chi)=0, \chi=c y$, and Eq. (28) has the solution

$$
\psi=\eta(y) \exp (\beta x)-\frac{c y}{d_{G}}
$$

where $\eta(y)$ is an unknown function and $\beta=d_{G}^{2} /(c \operatorname{Re})$. Inserting Eq. (33) into (32) yields a single constraint equation:

$$
\eta^{\prime \prime}+\left(d_{G}+\beta^{2}\right) \eta=0
$$

The solution is

$$
\eta(y)=c_{s} \sin \left(\sqrt{d_{G}+\beta^{2}} y\right)+c_{c} \cos \left(\sqrt{d_{G}+\beta^{2}} y\right)
$$

Choosing the forms of the coefficients $\sqrt{d_{G}+\beta^{2}}=\lambda, c_{s}=A / \lambda$, $c_{c}=0, c=-d_{G}$, and $d_{G}=-\operatorname{Re} \beta$ gives the classical Kovasznay flow [24]

$$
\psi=y-\frac{A}{\lambda} \sin (\lambda y) \exp (\beta x) \quad \beta=-\frac{1}{2}\left[\sqrt{\mathrm{Re}^{2}+4 \lambda^{2}}-\mathrm{Re}\right]
$$

where $A$ and $\lambda$ are arbitrary constants. A representative flow is shown in Fig. 1.

If the same coefficients are chosen as the Kovasznay solution except $c_{s}=-A i / \lambda, \quad c_{c}=-A / \lambda$, and $\sqrt{d_{G}+\beta^{2}}=-i \lambda$, Lin and

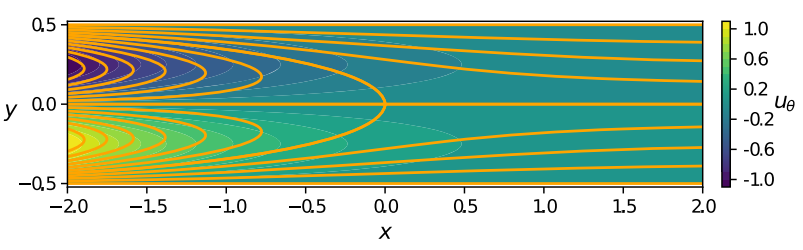

Fig. 1 Kovasznay flow with $\operatorname{Re}=40, A=1, \lambda=2 \pi$, and $d_{f}=1$. The streamlines are those of the in-plane velocity field, and contours are of the out-of-plane velocity component.

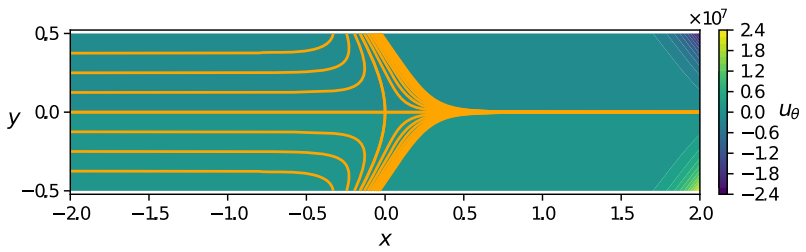

Fig. 2 Wang's flow (Eq. (35)) at $\operatorname{Re}=13, A=1, \lambda=2 \pi$, and $d_{f}=1$. The streamlines are those of the in-plane velocity field, and contours are of the out-of-plane velocity component.

Tobak's solution may be obtained [29]:

$$
\begin{aligned}
& \psi=y-\frac{A}{\lambda} \exp (\lambda y+\beta x) \\
& \beta=\frac{1}{2}\left[\sqrt{\operatorname{Re}^{2}-4 \lambda^{2}}+\operatorname{Re}\right]
\end{aligned}
$$

Wang's solution appears when the coefficients of Lin and Tobak's solution are selected, with the exception $c_{c}=0$ [19]

$$
\begin{aligned}
& \psi=y-\frac{A}{\lambda} \sinh (\lambda y) \exp (\beta x) \\
& \beta=\frac{1}{2}\left[\sqrt{\operatorname{Re}^{2}-4 \lambda^{2}}+\operatorname{Re}\right]
\end{aligned}
$$

This flow is shown in Fig. 2.

Aside from the above solutions, many other solutions have been obtained using the extended Beltrami method, working in Cartesian coordinates. Early solutions have been reviewed by Wang [2,3] and Drazin and Riley [4]. More recently, Chandna and Oku-Ukpong [30] have obtained solutions by assuming polynomial forms of $\chi(x, y)$, which were later generalized by Islam et al. [31]. Hui [32] has retained the unsteady terms in their formulation and arrived at a variety of solutions exponentially dependent on time. Jamil [33] assumed $\chi=-U \exp (a x+b y)$ and found solutions depending only on $a x+b y$, indicating the flow is unidirectional.

4.2 Plane Polar Coordinates. Working in polar coordinates $(r, \theta, z)$, we have $h_{1}=1$ and $h_{2}=r$. If we assume $\chi=-a\left(d_{G} r^{2}+\right.$ 4), a general solution for $\psi$ may be found using Eq. (28):

$$
\psi=a r^{2}+\eta(r) \exp \left(\frac{d_{G} \theta}{2 a \mathrm{Re}}\right)
$$

where $\eta(r)$ is an unknown function. Inserting Eq. (36) into Eq. (29) yields

$$
\eta^{\prime \prime}+\frac{\eta^{\prime}}{r}+d_{G}\left(1+\frac{d_{G}^{3}}{4 a^{2} \operatorname{Re}^{2} r^{2}}\right) \eta=0
$$


whose solution is

$$
\eta(r)=c_{\eta 1} J_{\frac{i d_{G}}{a \mathrm{Re}}}\left(\sqrt{d_{G}} r\right)+c_{\eta 2} Y_{\frac{i d_{G}}{a \mathrm{Re}}}\left(\sqrt{d_{G}} r\right)
$$

where $J_{n}(x)$ and $Y_{n}(x)$ are Bessel functions of order $n$ of the first and second kinds, respectively. Note that the orders of each of the Bessel functions in Eq. (38) are imaginary. To eliminate the imaginary parts, we will leverage the identity $\operatorname{Im}\left\{J_{i v}(x)\right\} \approx$ $\tanh (\pi v / 2) \operatorname{Re}\left\{Y_{i v}(x)\right\}$ for small $x$ [34] by choosing $c_{\eta 2}=-c_{\eta 1}$ $\tanh \left(\pi d_{G} /(2 a \operatorname{Re})\right) i$. Noting that $\operatorname{Re}\left\{J_{i v}(x)\right\} \propto \operatorname{Im}\left\{Y_{i v}(x)\right\}$, the final solution may be written as

$$
\psi=a r^{2}+c_{\eta} \operatorname{Re}\left\{J_{\frac{i d_{G}}{a \mathrm{Re}}}\left(\sqrt{d_{G}} r\right)\right\} \exp \left(\frac{d_{G} \theta}{2 a \operatorname{Re}}\right)
$$

Since the exponential term containing $\theta$ cannot be made cyclic without introducing further imaginary components, Eq. (39) has limited utility since it may only be applied across a finite range of $\theta$, where suitable boundary conditions must be applied at the domain boundaries (upper and lower bounds of $\theta$ ).

\section{3D Flows in Extruded Coordinate Systems}

We now shift the focus to 3D fluid motions in extruded coordinate systems $\left(h_{3}=1\right)$, where Eqs. (26) and (27) reduce to

$$
\begin{aligned}
& K(\phi, \psi)=\frac{1}{\operatorname{Re}}\left\{\nabla^{2} \phi-d_{F} V\left(d_{H} \psi+\chi\right)\right\} \\
& K(\chi, \psi)=\frac{1}{\operatorname{Re}}\left\{\nabla^{2} \chi-d_{H} V\left(d_{H} \psi+\chi\right)\right\}
\end{aligned}
$$

while the vorticity definition (25) becomes

$$
\xi=d_{H} \psi+\chi=-\frac{D^{2}(\psi)}{V}
$$

where $d_{H}=d_{F}^{2}+d_{G}$. One can immediately recognize that solutions to the 2D planar equations of Sec. 4 are also solutions to Eqs. (41) and (42), where $d_{G}$ is relabeled $d_{H}$ and the cross-flow, $u_{3}$, may be constructed from the solution to Eq. (40). In all cases, the function $\phi=d_{F} \chi / d_{H}$ is admissible, such that Eqs. (40) and (41) are linearly dependant. As an example, Kovasznay's flow (Eq. (33)) supports a cross-flow

$$
u_{z}=\sigma=d_{F} \psi+\phi=-\frac{d_{F} A}{\lambda} \sin (\lambda y) \exp (\beta x)
$$

The cross-flow variation is shown in the color contours in Fig. 1. A similar cross-flow solution may be constructed for Wang's solution (Eq. (35)) and the cross-flow component has been included in Fig. 2.

\section{Swirling Flow in Cylindrical Coordinates}

In cylindrical coordinates $(z, r, \theta)$, the scale coefficients are $h_{1}=$ $h_{2}=1$ and $h_{3}=r$. Note that the present coordinate system is a permutation of the plane polar coordinate system in Sec. 4.2; in that section, we studied motions in the $r-\theta$ plane where no axial variation is permitted. Here, we study flows which vary in the meridional $(z-r)$ plane but are invariant with respect to $\theta$. Equations (25)-(27) become

$$
\begin{gathered}
\xi=\frac{d_{F}^{2}}{r^{2}} \psi+d_{G} \psi+\chi=-\frac{D^{2}(\psi)}{r} \\
K(\phi, \psi)=\frac{1}{\operatorname{Re}}\left\{r^{2} D^{2}(\phi)-r d_{F}\left(d_{F}^{2} \psi+d_{G} r^{2} \psi+r^{2} \chi\right)\right\}
\end{gathered}
$$

$$
\begin{aligned}
& K(\chi, \psi)-\frac{2}{r^{3}}\left[\left(d_{F} \psi+\phi\right) \frac{\partial \phi}{\partial z}+d_{F} \phi \frac{\partial \psi}{\partial z}\right] \\
& =\frac{1}{\operatorname{Re}}\left\{-r\left(d_{F}^{2}+d_{G} r^{2}\right)\left(\frac{d_{F}^{2}}{r^{2}} \psi+d_{G} \psi+\chi\right)+D^{2}\left(r^{2} \chi\right)+4 d_{G} \frac{\partial \psi}{\partial r}\right\}
\end{aligned}
$$

6.1 Vanishing Centrifugal Term. The second term in Eq. (46) is the centrifugal acceleration term; it represents the influence of angular momentum variation along the $z$ axis on the motion in the meridional plane. In this subsection, we will discuss a family of solutions which have a vanishing centrifugal term. To facilitate this, we begin with the assumptions $d_{F}=0$ and $\phi=\phi(r)$. Equation (45) reduces to

$$
-\frac{d \phi}{d r} \frac{\partial \psi}{\partial z}=\frac{r^{2} D^{2}(\phi)}{\operatorname{Re}}
$$

Since $\psi$ is the only $z$-dependent term in Eq. (47), we must also have

$$
\psi=z \Psi(r)+\delta(r)
$$

to obtain non-trivial solutions. Inserting this corollary into Eq. (44) yields

$$
\chi=\frac{z}{r^{3}}\left(-d_{G} r^{3} \Psi-r \frac{d^{2} \Psi}{d r^{2}}+\frac{d \Psi}{d r}\right)+\frac{1}{r^{3}}\left(-d_{G} r^{3} \delta-r \frac{d^{2} \delta}{d r^{2}}+\frac{d \delta}{d r}\right)
$$

Furthermore, $\chi$ may be broken into two terms to accommodate Eq. (49):

$$
\chi=z X(r)+Y(r)
$$

Equation (49) can be broken into two equations to balance the coefficients of the $z$ terms:

$$
\begin{gathered}
X(r)=\frac{1}{r^{3}}\left(-d_{G} r^{3} \Psi-r \frac{d^{2} \Psi}{d r^{2}}+\frac{d \Psi}{d r}\right) \\
Y(r)=\frac{1}{r^{3}}\left(-d_{G} r^{3} \delta-r \frac{d^{2} \delta}{d r^{2}}+\frac{d \delta}{d r}\right)
\end{gathered}
$$

Using these reduced forms, Eq. (46) can be simplified with the help of MAPLE ${ }^{\mathrm{TM}}$ software [35]:

$$
\begin{gathered}
\zeta \Psi^{\prime \prime \prime}+\Psi^{\prime \prime}+\frac{\operatorname{Re}}{2}\left(\Psi \Psi^{\prime \prime}-\Psi^{\prime 2}\right)=C \\
\zeta \delta^{\prime \prime \prime}+\delta^{\prime \prime}+\frac{\operatorname{Re}}{2}\left(\Psi \delta^{\prime \prime}-\Psi^{\prime} \delta^{\prime}\right)=D \\
\zeta=\frac{k \operatorname{Re} r^{2}}{2}
\end{gathered}
$$

where ' denotes differentiation with respect to $\zeta$. Equations (53) and (54) are a system of ordinary differential equations in $\zeta$ and qualify as a family of exact solutions under the criteria described in the introduction. Terrill and Thomas [36] have studied the case where $\delta=\Psi$ numerically with a constant suction/injection velocity at $\zeta=1$, finding four solution branches. While Terrill and Thomas considered only $\operatorname{Re}$ and $C$ as free parameters, the general case also requires choosing $D$, along with six additional parameters to fully define the boundary conditions. We will not describe this rich set of solutions here but analyze one special case, appearing when we assume the function

$$
\Psi=\frac{2}{\operatorname{Re}} \zeta
$$


which satisfies Eq. (53). Using this result, solving Eq. (54) (using MAPLE $^{\text {TM }}$ software) yields

$$
\delta=c_{1}+c_{2}\left(\frac{\zeta^{2}}{2}+\zeta\right)-D \zeta+\frac{c_{3}}{2}\left(\zeta[\zeta+2] \mathrm{E}_{1}(\zeta)-[\zeta+1] \exp (-\zeta)\right)
$$

where $\mathrm{E}_{1}(x)=-\int_{-x}^{\infty} \exp (s) / s d s$ is the exponential integral. We can set $c_{1}=0$ and $D=c_{2}$, without loss of generality. Furthermore, Eq. (47) is used to evaluate $\phi$ and the solution is

$$
\begin{gathered}
\psi=\frac{2}{\operatorname{Re}} \zeta z+\frac{c_{2}}{2} \zeta^{2}+\frac{c_{3}}{2}\left(\zeta[\zeta+2] \mathrm{E}_{1}(\zeta)-[\zeta+1] \exp (-\zeta)\right) \\
u_{\theta}=\frac{\phi}{r}=\frac{\Gamma}{2 \pi r}(1-\exp (-\zeta))
\end{gathered}
$$

When $\delta=0$, the well-known Burgers vortex [37] is recovered. If we instead retain $c_{2}$ and set $c_{3}=0$, Berker's solution is obtained [14], which describes two opposing streams with a stagnation point at the origin. We find that Berker's solution supports the same circumferential velocity profile as Burgers vortex, as shown by the present analysis. Interestingly, the parameter $d_{G}$ does not appear in the final solution and has no bearing on the velocity field.

Other researchers have developed generalizations of the Burgers vortex. Sullivan [38] discovered that the ansatz $\Psi=2 \zeta / \operatorname{Re}+$ $A(1-\exp (\beta \zeta))$ produces a solution describing a two-cell vortex (which the present formulation also admits), wherein the flow within a bounding radius recirculates along the $z$-axis while flow outside the bounding radius recirculates away from the axis. Bellamy-Knights [39] has generalized Sullivan's solution to include unsteady motions of the bounding surface. More recently, Craik [40] has thoroughly explored all solutions of the type $\Psi=$ $A r^{2}+B \log r$, where $A$ and $B$ are constants or functions of time. When $B=0$, many unsteady generalizations of Burgers vortex may be found. The solution described by Eqs. (57) and (58) with $c_{3} \neq 0$ is an extension to Burger's solution which has not yet been considered; however, the axial velocity becomes singular at $r=0$, due to the presence of $\mathrm{E}_{1}(\zeta)$. Consequently, it appears to have little practical use.

6.2 Swirling Kovasznay Flow. Another solution may be found by assuming that the auxiliary functions vary only with $r$; $\phi=\phi(r)$ and $\chi=\chi(r)$. With these guesses, solving Eq. (45) returns:

$$
\begin{gathered}
\psi=\eta(r) \exp (\beta(r) z)+\kappa(r) \\
\beta(r)=\frac{d_{F} r}{\operatorname{Re} \phi^{\prime}}\left(d_{G} r^{2}+d_{F}^{2}\right) \\
\kappa(r)=\frac{\phi^{\prime \prime}-\left(\phi^{\prime} / r\right)-d_{F} r^{2} \chi}{d_{F}\left(d_{F}^{2}+d_{G} r^{2}\right)}
\end{gathered}
$$

where $\eta(r)$ is an unknown function and ' denotes differentiation with respect to $r$. Inserting Eq. (59) into Eq. (44) yields

$$
\begin{aligned}
& \left(d_{G} r^{2}+d_{F}^{2}\right)(\eta \exp (\beta z)+\kappa)+r^{2} \chi \\
& =\left(z \eta \frac{\beta^{\prime}}{r}+\frac{\eta^{\prime}}{r}-z^{2} \eta\left(\beta^{\prime}\right)^{2}-z \eta \beta^{\prime \prime}-2 z \eta^{\prime} \beta^{\prime}-\beta^{2} \eta-\eta^{\prime \prime}\right) \\
& \quad \times \exp (\beta z)-\kappa^{\prime \prime}+\frac{\kappa^{\prime}}{r}
\end{aligned}
$$

Since Eq. (62) contains no unknown functions of $z$, the coefficients of the terms containing $z$ must sum to 0 :

$$
\exp (\beta z): \eta^{\prime \prime}-\frac{\eta^{\prime}}{r}+\left(d_{G} r^{2}+d_{F}^{2}+\beta^{2}\right) \eta=0
$$

$$
\begin{gathered}
z \exp (\beta z): 2 \eta^{\prime} \beta^{\prime}+\eta \beta^{\prime \prime}-\frac{\eta}{r} \beta^{\prime}=0 \\
z^{2} \exp (\beta z): \eta\left(\beta^{\prime}\right)^{2}=0 \\
z^{0}: \kappa^{\prime \prime}-\frac{\kappa^{\prime}}{r}+\left(d_{G} r^{2}+d_{F}^{2}\right) \kappa+r^{2} \chi=0
\end{gathered}
$$

Equation (65) is only satisfied when $\eta=0$ or $\beta=$ const. Taking the latter solution, Eq. (64) is immediately satisfied, and $\phi$ may be found using (60):

$$
\phi=\frac{d_{F} r^{2}}{4 \operatorname{Re} \beta}\left(d_{G} r^{2}+2 d_{F}^{2}\right)
$$

where an integration constant has been set to 0 to avoid singular solutions of $u_{\theta}$ at $r=0$. Equation (66) may be solved using Eqs. (61) and (67):

$$
\chi=\left(d_{G} r^{2}+d_{F}^{2}\right)\left(\frac{c_{\chi 1}}{r^{2}}+c_{\chi 2}\right)+\frac{d_{G}^{3} r^{6}+d_{F}^{6}-8 d_{F}^{2} d_{G}}{4 d_{G} \operatorname{Re} \beta r^{2}}
$$

Substituting the above results into Eq. (44) yields (after much simplification)

$$
\begin{aligned}
& \left(4 \operatorname{Re} c_{\chi 2} \beta d_{G}^{2} r^{4}-3 d_{F}^{2} d_{G}^{2} r^{4}-4 \operatorname{Re} c_{\chi 1} \beta d_{F}^{2} d_{G}-d_{F}^{6}+8 d_{F}^{2} d_{G}\right) \eta \\
& \quad+d_{G}^{2} r^{3} \eta^{\prime}=0
\end{aligned}
$$

Equations (63) and (69) are incompatible, and one must be eliminated to yield an admissible solution. A non-trivial solution arises when $d_{G}=0$ and $c_{\chi 1}=\left(8-d_{F}^{4} / d_{G}\right) /(4 \operatorname{Re} \beta)$, so Eq. (69) is satisfied. Then, Eq. (63) may be solved:

$$
\eta=c_{\eta 1} r J_{1}(\lambda r)+c_{\eta 2} r Y_{1}(\lambda r)
$$

where $\lambda=\sqrt{d_{F}^{2}+\beta^{2}}$, and $J_{n}$ and $Y_{n}$ are $n^{\text {th }}$-order Bessel functions of the first and second kinds, respectively. Choosing $c_{\eta 2}=0$ eliminates the singularity at the axis produced by $Y_{1}(0)$. The final solution reads

$$
\begin{array}{r}
\psi=c\left[r^{2}-\frac{2}{\lambda} r J_{1}(\lambda r) \exp (\beta z)\right] \\
\sigma=\Omega r^{2}-\frac{2 c d_{F} r}{\lambda} J_{1}(\lambda r) \exp (\beta z) \\
\beta=\frac{d_{F}^{3}}{2 \operatorname{Re}_{\theta}}, \quad d_{F}=-\sqrt{\sqrt[3]{\frac{2}{9}} b-\sqrt[3]{\frac{32}{b}}}, \\
b=\operatorname{Re}_{\theta}^{2}\left(9 \lambda^{2}+\sqrt{3\left(16 \operatorname{Re}_{\theta}^{2}+27 \lambda^{4}\right)}\right)
\end{array}
$$

where setting $c_{\eta 1}=2 c / \lambda$ shifts the stagnation point to the origin and $c=-c_{\chi 2}$. The Reynolds number has been replaced by $\operatorname{Re}_{\theta}=\operatorname{Re} \Omega^{*}$ and $\Omega=\Omega^{*}-c d_{F}$. A representative flow is shown in Fig. 3 and two $3 \mathrm{D}$ streamlines showing the recirculating and nonrecirculating motions of the particles near the axis are visible in Fig. 4. These streamlines bear a strong resemblance to streamlines produced from computational fluid dynamics simulation results (see, for example, Figs. 14 and 15 in Ref. [41]), although the present flow is incompressible whereas the flow inside the Ranque-Hilsch vortex tube is highly compressible. To the best of our knowledge, the solution described by Eqs. (71) and (72) is new, although it may be considered a generalization of the solution presented by Fatsis et al. [42]. The solution set may be viewed as the cylindrical analogue of Kovasznay's solution, as both flows are cellular, have a uniform base flow, and contain strong recirculation regions. The clear difference is that the above solution collapses to a uniform flow along the axis when the swirl vanishes, 


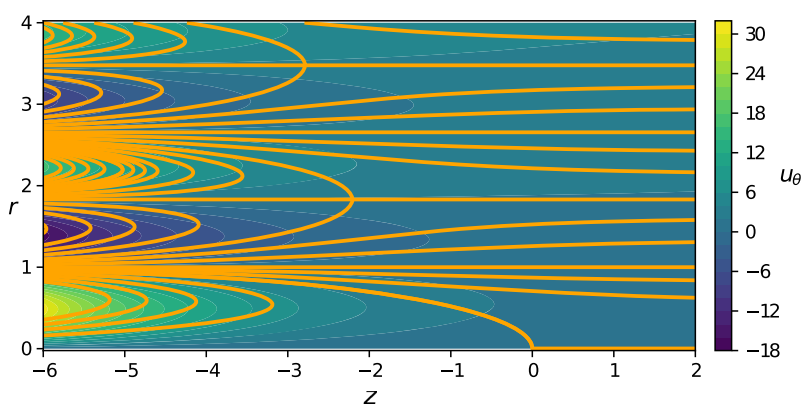

Fig. 3 The swirling flow solution given by Eqs. (71) and (72) with $\operatorname{Re}_{\theta}=50, \lambda=j_{0}, \Omega=1$, and $c=1$ where $j_{0}$ is the first root of $J_{1}(x)$. The streamlines are those of the meridional plane velocity field, and the color contours correspond to the circumferential velocity distribution.

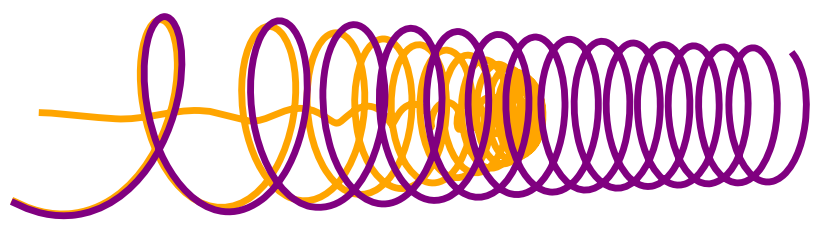

Fig. 4 3D streamlines of the solution given by Eqs. (71) and (72) with $\operatorname{Re}_{\theta}=50, \lambda=j_{0}, \Omega=40$, and $c=1$ where $j_{0}$ is the first root of $J_{1}(x)$. The orange streamline $(\psi=-0.01872)$ recirculates back along the axis, while the purple streamline $(\psi=0.313919)$ proceeds along the axis.

whereas the Kovasznay solution has no such dependence on the out-of-plane velocity. Since the sign of the Bessel function alternates between cells, the sign of $u_{\theta}$ may become negative in the initial even numbered cells (assuming the cells are numbered sequentially starting with the innermost cell) depending on whether the first or second term in Eq. (72) dominates, meaning the bulk of the fluid in these cells can rotate in an opposite sense to its neighbors about the $z$-axis.

As shown in Fig. 5, increasing $\operatorname{Re}_{\theta}$ causes the recirculating regions to become increasingly slender, a behavior also exhibited by Kovasznay flow. Furthermore, the swirl magnitudes near the origin decrease with increasing $\operatorname{Re}_{\theta}$. Finally, the adjustment of the swirl factor $\Omega$ changes the magnitude of the "background" rigid body swirl, and the circumferential velocity is increased with

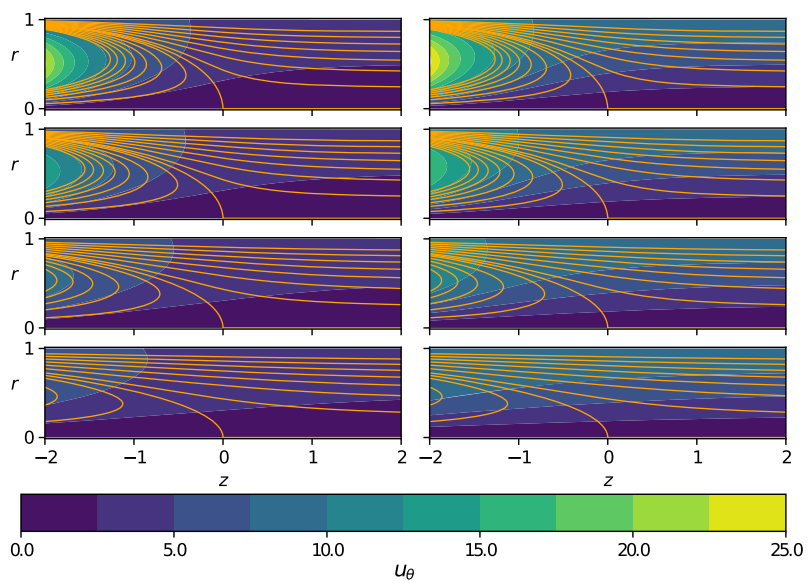

Fig. 5 Plots are described by the caption of Fig. 3 except $\mathbf{R e}_{\theta}=$ $5,10,20,50$ for each row and $\Omega=1,5$ for each column increasing radius; however, it has no influence on the streamlines in the meridional plane.

While the counter-rotating annular cells visible in Fig. 3 are certainly interesting, the flow within the innermost cell is the most readily applicable to the study of various fluid machinery. First, the present solution might be applied to the study of cyclonic separators, which are frequently analyzed using exact techniques; however, viscous effects have often been neglected in the search for exact solutions (cf. Ref. [43]). In a related application, Maicke et al. [44] have numerically solved the axisymmetric Euler equations to study compressible flows in a bi-directional vortex, where swirling fluid is injected near the periphery of a tube and is withdrawn near the axis at the same axial location. An important finding of Maicke et al. is that for high swirl flows, compressibility appears to have little influence on the solution field. The present incompressible solution may be readily applied to their problem when $\operatorname{Re}_{\theta}$ is small. Finally, the present solution bears strong similarities to axisymmetric Ranque-Hilsch flow. Most importantly, it includes the effect of viscous forces, which has been shown to be the dominant energy transfer mechanism across the stagnation streamline $[45,46]$.

\section{Summary and Discussion}

In the present work, the extended Beltrami approach first presented by Wang [19] has been reviewed and extended. We have shown that our general formulation (Eqs. (25)-(27)) can be used to analyze viscous three-dimensional flows in planar and revolved coordinate systems. Specifically, we are able to recover all known 2D planar solutions and have discovered that each one supports an out-of-plane velocity distribution which is closely related to the streamfunction solution. Furthermore, we have analyzed the equations of motion in cylindrical coordinates with a vanishing centrifugal term, finding a new generalization of Burger's vortex. Finally, we have discovered a new swirling flow solution describing flow resembling Kovasznay flow and also bears strong similarities to Ranque-Hilsch flow.

Comparing the planar results of Secs. 4 and 5 with the axisymmetric flows of Sec. 6, we find that the planar solutions can be separated into the in-plane and out-of-plane motions, where the in-plane velocities are unaffected by the out-of-plane motion. This is because the centrifugal term, given by $K\left(\sigma / h_{3}^{2}, \sigma\right)$, vanishes for planar flows, and only a one-way coupling exists between the vorticity and out-of-plane momentum equations. The centrifugal term does not vanish, in general, for axisymmetric flows because $h_{3} \neq 1$. If the swirl velocity is independent of $z$, however, the equations describe an important sub-class of flows in which the meridional plane motion is independent of the swirl, some of which are described in Sec. 6.1. In contrast, the swirling flow solution given by Eqs. (71) and (72) appears to be the first of its kind, in that it is a $3 \mathrm{D}$ axisymmetric Navier-Stokes solution with a non-vanishing centrifugal term.

\section{Acknowledgment}

The authors gratefully acknowledge the financial support from the Natural Sciences and Engineering Research Council of Canada (NSERC; Funder ID: 10.13039/501100000038).

\section{Appendix: Derivation of the Working Equations}

Here, we aim to develop the third components of the momentum and vorticity (Eqs. (2) and (3), respectively) in terms of $\sigma=h_{3} u_{3}$ and $\xi=\omega_{3} / h_{3}$. Beginning with Eq. (2), the transient term may be written as

$$
\frac{\partial \mathbf{u}}{\partial t}=\frac{1}{h_{2} h_{3}} \frac{\partial^{2} \psi}{\partial x_{2} \partial t} \hat{\mathbf{e}}_{1}-\frac{1}{h_{1} h_{3}} \frac{\partial^{2} \psi}{\partial x_{1} \partial t} \hat{\mathbf{e}}_{2}+\frac{\partial u_{3}}{\partial t} \hat{\mathbf{e}}_{3}
$$


The advection term is given by

$$
\begin{aligned}
(\mathbf{u} \cdot \nabla) \mathbf{u}= & \nabla\left(\frac{\mathbf{u} \cdot \mathbf{u}}{2}\right)+\omega \times \mathbf{u}=\nabla\left(\frac{\mathbf{u} \cdot \mathbf{u}}{2}\right)+\frac{1}{h_{1}}\left[\xi \frac{\partial \psi}{\partial x_{1}}-\frac{\sigma}{h_{3}^{2}} \frac{\partial \sigma}{\partial x_{1}}\right] \hat{\mathbf{e}}_{1}+\frac{1}{h_{2}}\left[\xi \frac{\partial \psi}{\partial x_{2}}-\frac{\sigma}{h_{3}^{2}} \frac{\partial \sigma}{\partial x_{2}}\right] \hat{\mathbf{e}}_{2} \\
& +\frac{1}{h_{1} h_{2} h_{3}^{2}}\left(\frac{\partial \sigma}{\partial x_{1}} \frac{\partial \psi}{\partial x_{2}}-\frac{\partial \sigma}{\partial x_{2}} \frac{\partial \psi}{\partial x_{1}}\right) \hat{\mathbf{e}}_{3}=\nabla\left(\frac{\mathbf{u} \cdot \mathbf{u}}{2}\right)+\frac{1}{h_{1}}\left[\xi \frac{\partial \psi}{\partial x_{1}}-\frac{\sigma}{h_{3}^{2}} \frac{\partial \sigma}{\partial x_{1}}\right] \hat{\mathbf{e}}_{1}+\frac{1}{h_{2}}\left[\xi \frac{\partial \psi}{\partial x_{2}}-\frac{\sigma}{h_{3}^{2}} \frac{\partial \sigma}{\partial x_{2}}\right] \hat{\mathbf{e}}_{2}+\frac{K(\sigma, \psi)}{h_{3} V} \hat{\mathbf{e}}_{3}
\end{aligned}
$$

The pressure term is written as

The viscous term may be written as

$$
-\nabla p=-\frac{1}{h_{1}} \frac{\partial p}{\partial x_{1}} \hat{\mathbf{e}}_{1}-\frac{1}{h_{2}} \frac{\partial p}{\partial x_{2}} \hat{\mathbf{e}}_{2}
$$

$$
\begin{aligned}
\frac{\nabla^{2} \mathbf{u}}{\operatorname{Re}}=-\frac{\nabla \times \omega}{\operatorname{Re}} & =\frac{1}{\operatorname{Re} V}\left(-h_{1} \frac{\partial\left(h_{3} \omega_{3}\right)}{\partial x_{2}} \hat{\mathbf{e}}_{1}+h_{2} \frac{\partial\left(h_{3} \omega_{3}\right)}{\partial x_{1}} \hat{\mathbf{e}}_{2}+h_{3}\left[\frac{\partial\left(h_{1} \omega_{1}\right)}{\partial x_{2}}-\frac{\partial\left(h_{2} \omega_{2}\right)}{\partial x_{1}}\right] \hat{\mathbf{e}}_{3}\right) \\
& =-\frac{h_{1}}{\operatorname{Re} V} \frac{\partial\left(h_{3}^{2} \xi\right)}{\partial x_{2}} \hat{\mathbf{e}}_{1}+\frac{h_{2}}{\operatorname{Re} V} \frac{\partial\left(h_{3}^{2} \xi\right)}{\partial x_{1}} \hat{\mathbf{e}}_{2}+\frac{h_{3}}{\operatorname{Re} V} D^{2}(\sigma) \hat{\mathbf{e}}_{3}
\end{aligned}
$$

Re-assembling the $\hat{\mathbf{e}}_{3}$ components of Eqs. (A1)-(A4) and multiplying by $h_{3} V$ yields Eq. (9). The vorticity equation terms are similarly developed here. The transient term is the time derivative of Eq. (7)

$$
\frac{\partial \omega}{\partial t}=\frac{1}{h_{2} h_{3}} \frac{\partial^{2} \sigma}{\partial x_{2} \partial t} \hat{\mathbf{e}}_{1}-\frac{1}{h_{1} h_{3}} \frac{\partial^{2} \sigma}{\partial x_{1} \partial t} \hat{\mathbf{e}}_{1}+h_{3} \frac{\partial \xi}{\partial t} \hat{\mathbf{e}}_{3}
$$

Taking the curl of (A2) gives the vorticity equation advection term

$$
\begin{aligned}
\nabla \times(\omega \times \mathbf{u})= & \frac{h_{1}}{V}\left[\frac{\partial}{\partial x_{2}}\left(\frac{K(\sigma, \psi)}{V}\right)\right] \hat{\mathbf{e}}_{1}-\frac{h_{2}}{V}\left[\frac{\partial}{\partial x_{1}}\left(\frac{K(\sigma, \psi)}{V}\right)\right] \hat{\mathbf{e}}_{2}+\frac{h_{3}}{V}\left[\frac{\partial}{\partial x_{1}}\left(\xi \frac{\partial \psi}{\partial x_{2}}-\frac{\sigma}{h_{3}^{2}} \frac{\partial \sigma}{\partial x_{2}}\right)-\frac{\partial}{\partial x_{2}}\left(\xi \frac{\partial \psi}{\partial x_{1}}-\frac{\sigma}{h_{3}^{2}} \frac{\partial \sigma}{\partial x_{1}}\right)\right] \hat{\mathbf{e}}_{3} \\
& =\frac{h_{1}}{V}\left[\frac{\partial}{\partial x_{2}}\left(\frac{K(\sigma, \psi)}{V}\right)\right] \hat{\mathbf{e}}_{1}-\frac{h_{2}}{V}\left[\frac{\partial}{\partial x_{1}}\left(\frac{K(\sigma, \psi)}{V}\right)\right] \hat{\mathbf{e}}_{2}+\frac{h_{3}}{V}\left[K(\xi, \psi)-K\left(\frac{\sigma}{h_{3}^{2}}, \sigma\right)\right] \hat{\mathbf{e}}_{3}
\end{aligned}
$$

Since the curl of term (A3) is 0 , the pressure does not appear in the vorticity equation. Finally, taking the curl of Eq. (A4) gives the viscous terms

$$
\begin{aligned}
-\frac{\nabla \times(\nabla \times \omega)}{\operatorname{Re}} & =\frac{h_{1}}{\operatorname{Re} V}\left[\frac{\partial}{\partial x_{2}}\left(\frac{h_{3}^{2}}{V} D^{2}(\sigma)\right)\right] \hat{\mathbf{e}}_{1}-\frac{h_{2}}{\operatorname{Re} V}\left[\frac{\partial}{\partial x_{1}}\left(\frac{h_{3}^{2}}{V} D^{2}(\sigma)\right)\right] \hat{\mathbf{e}}_{2}+\frac{h_{3}}{\operatorname{Re} V}\left(\frac{\partial}{\partial x_{1}}\left[\frac{h_{2}}{h_{1} h_{3}} \frac{\partial\left(h_{3}^{2} \xi\right)}{\partial x_{1}}\right]+\frac{\partial}{\partial x_{2}}\left[\frac{h_{1}}{h_{2} h_{3}} \frac{\partial\left(h_{3}^{2} \xi\right)}{\partial x_{2}}\right]\right) \hat{\mathbf{e}}_{3} \\
& =\frac{h_{1}}{\operatorname{Re} V}\left[\frac{\partial}{\partial x_{2}}\left(\frac{h_{3}^{2}}{V} D^{2}(\sigma)\right)\right] \hat{\mathbf{e}}_{1}-\frac{h_{2}}{\operatorname{Re} V}\left[\frac{\partial}{\partial x_{1}}\left(\frac{h_{3}^{2}}{V} D^{2}(\sigma)\right)\right] \hat{\mathbf{e}}_{2}+\frac{h_{3}}{\operatorname{Re} V} D^{2}\left(h_{3}^{2} \xi\right) \hat{\mathbf{e}}_{3}
\end{aligned}
$$

Re-assembling the $\hat{\mathbf{e}}_{3}$ components of Eqs. (A5)-(A7) and multiplying by $V / h_{3}$ yields Eq. (10). While the $\hat{\mathbf{e}}_{1}$ and $\hat{\mathbf{e}}_{2}$ terms may also be assembled into equations, both of these are linearly dependent on Eq. (9) and are therefore superfluous.

\section{References}

[1] Pozrikidis, C., 2011, Introduction to Theoretical and Computational Fluid Dynamics, 2nd ed., Oxford University Press, New York, NY.

[2] Wang, C.-Y., 1989, "Exact Solutions of the Unsteady Navier-Stokes Equations," ASME Appl. Mech. Rev., 42(11S), pp. S269-S282.

[3] Wang, C.-Y., 1991, "Exact Solutions of the Steady-State Navier-Stokes Equations," Annu. Rev. Fluid Mech., 23(1), pp. 159-177.

[4] Drazin, P. G., and Riley, N., 2006, The Navier-Stokes Equations: A Classification of Flows and Exact Solutions, 1st ed., Cambridge University Press, Cambridge.

[5] Polyanin, A. D., and Aristov, S. N., 2011, "A New Method for Constructing Exact Solutions to Three-Dimensional Navier-Stokes and Euler Equations," Theor. Found. Chem. Eng., 45(6), pp. 885-890.

[6] Polyanin, A. D., and Zhurov, A. I., 2016, "Functional and Generalized Separable Solutions to Unsteady Navier-Stokes Equations," Int. J. Non-Linear Mech., 79, pp. 88-98.

[7] Kumar, M., and Kumar, R., 2014, "On Some New Exact Solutions of Incompressible Steady State Navier-Stokes Equations,” Meccanica, 49(2), pp. 335-345.

[8] Tsien, H. S., 1943, "Symmetrical Joukowsky Airfoils in Shear Flow," Q. Appl. Math., 1(2), pp. 130-148.

[9] Wang, C.-Y., 1990, "Exact Solutions of the Navier-Stokes Equations-The Generalized Beltrami Flows, Review and Extension," Acta Mech., 81(1-2), pp. 69-74.
[10] Hill, M. J. M., 1894, "VI. On a Spherical Vortex," Phil. Trans. R. Soc. A: Math. Phys. Eng. Sci., 185(1), pp. 213-245.

[11] O'Brien, V., 1961, "Steady Spheroidal Vortices More Exact Solutions to the Navier-Stokes Equation," Q. Appl. Math., 19(2), pp. 163-168.

[12] Fujimoto, M., Science, S., Uehara, K., and Yanase, S., 2015, "Vortex Solutions of the Generalized Beltrami Flows to the Euler Equations," e-print arXiv:1501.05620.

[13] Joseph, S. P., 2018, "Polynomial Solutions and Other Exact Solutions of Axisymmetric Generalized Beltrami Flows," Acta Mech., 229(7), pp. 2737-2750.

[14] Berker, R., 1963, Intégration des équations du Mouvement D’un Fluide Visqueux Incompressible, Springer, Berlin.

[15] Terrill, R. M., 1982, "An Exact Solution for Flow in a Porous Pipe," Z. Angew. Math. Mech., 33(4), pp. 547-552.

[16] Saccomandi, G., 1994, "Some Exact Pseudo-Plane Solutions of the First Kind for the Navier-Stokes Equations," Z. Angew. Math. Mech., 45(6), pp. 978-985.

[17] Saccomandi, G., 1994, "Some Unsteady Exact Pseudo-Plane Solutions for the Navier-Stokes Equations," Meccanica, 29(3), pp. 261-269.

[18] Weinbaum, S., and O'Brien, V., 1967, "Exact Navier-Stokes Solutions Including Swirl and Cross Flow," Phys. Fluids, 10(7), pp. 1438-1447.

[19] Wang, C.-Y., 1966, "On a Class of Exact Solutions of the Navier-Stokes Equations,” ASME J. Appl. Mech., 33(3), pp. 696-698.

[20] Lopez, J. M., 1990, “Axisymmetric Vortex Breakdown Part 1. Confined Swirling Flow,” J. Fluid Mech., 221, p. 533.

[21] Polyanin, A. D., 2001, "Exact Solutions to the Navier-Stokes Equations With Generalized Separation of Variables," Dokl. Phys., 46(10), pp. 726-731.

[22] Lewellen, W. S., 1962, "A Solution for Three-Dimensional Vortex Flows With Strong Circulation," J. Fluid Mech., 14(3), pp. 420-432.

[23] Granger, R., 1966, "Steady Three-Dimensional Vortex Flow," J. Fluid Mech., 25(3), pp. 557-576.

[24] Kovasznay, L. I. G., 1948, "Laminar Flow Behind a Two-Dimensional Grid," Proc. Camb. Phil. Soc., 44(May), pp. 58-62. 
[25] Taylor, G., 1923, "On the Decay of Vortices in a Viscous Fluid," London Edinburgh Dublin Phil. Mag. J. Sci., 46(274), pp. 671-674.

[26] Leprovost, N., Dubrulle, B., and Chavanis, P. H., 2006, "Dynamics and Thermodynamics of Axisymmetric Flows: Theory," Phys. Rev. E: Stat Nonlinear Soft Matter Phys., 73(4), pp. 1-18.

[27] Batchelor, G. K., 1964, "Axial Flow in Trailing Line Vortices," J. Fluid Mech., 20(4), p. 645.

[28] Bhattacharya, S., 2007, "Exact Analytical Solutions for Steady Three-Dimensional Inviscid Vortical Flows," J. Fluid Mech., 590, pp. 147-162.

[29] Lin, S. P., and Tobak, M., 1986, "Reversed Flow Above a Plate With Suction," AIAA J., 24(2), pp. 334-335.

[30] Chandna, O. P., and Oku-Ukpong, E. O., 1994, "Flows for Chosen Vorticity Functions-Exact Solutions of the Navier-Stokes Equations," Int. J. Math. Math. Sci., 17(1), pp. 155-164.

[31] Islam, S., Zhou, C.-Y., and Ran, X.-J., 2008, "Exact Solutions for Different Vorticity Functions of Couple Stress Fluids," J. Zhejiang Univ. Sci. A, 9(5), pp. 672-680.

[32] Hui, W. H., 1987, "Exact Solutions of the Unsteady Two-Dimensional NavierStokes Equations," Z. Angew. Math. Mech., 38(5), pp. 689-702.

[33] Jamil, M., 2010, "A Class of Exact Solutions to Navier-Stokes Equations for the Given Vorticity Basic Governing Equations,” J. Nonlinear Sci., 9(3), pp. 296-304.

[34] Dunster, T. M., 1990, "Bessel Functions of Purely Imaginary Order, With an Application to Second-Order Linear Differential Equations Having a Large Parameter," SIAM J. Math. Anal., 21(4), pp. 995-1018.

[35] Maplesoft: A Division of Waterloo Maple Inc., 2018, Maple 2018.1.

[36] Terrill, R. M., and Thomas, P. W., 1969, "On Laminar Flow Through a Uniformly Porous Pipe," Appl. Sci. Res., 21(1), pp. 37-67.
[37] Burgers, J., 1948, “A Mathematical Model Illustrating the Theory of Turbulence," Adv. Appl. Mech., 1(1), pp. 171-199.

[38] Sullivan, R. D., 1959, "A Two-Cell Vortex Solution of the Navier-Stokes Equations," J. Aerospace Sci., 26(11), pp. 767-768.

[39] Bellamy-Knights, P. G., 1970, "An Unsteady Two-Cell Vortex Solution of the Navier-Stokes Equations,” J. Fluid Mech., 41(3), pp. 673-687.

[40] Craik, A. D. D., 2009, "Exact Vortex Solutions of the Navier-Stokes Equations With Axisymmetric Strain and Suction or Injection," J. Fluid Mech., 626, pp. 291-306.

[41] Farouk, T., and Farouk, B., 2007, "Large Eddy Simulations of the Flow Field and Temperature Separation in the Ranque-Hilsch Vortex Tube,” Int. J. Heat Mass Transfer, 50(23-24), pp. 4724-4735.

[42] Fatsis, A., Statharas, J., Panoutsopoulou, A., and Vlachakis, N., 2010, “A New Class of Exact Solutions of the Navier-Stokes Equations for Swirling Flows in Porous and Rotating Pipes," WIT Trans. Eng. Sci., 69, pp. 67-78.

[43] Bloor, M. I. G., and Ingham, D. B., 1987, "The Flow in Industrial Cyclones," J. Fluid Mech., 178(1987), p. 507.

[44] Maicke, B. A., Cecil, O. M., and Majdalani, J., 2017, "On the Compressible Bidirectional Vortex in a Cyclonically Driven Trkalian Flow Field," J. Fluid Mech., 823, pp. 755-786.

[45] Aljuwayhel, N. F., Nellis, G. F., and Klein, S. A., 2005, "Parametric and Internal Study of the Vortex Tube Using a CFD Model," Int. J. Refrig., 28(3), pp. $442-450$.

[46] Dyck, N. J., and Straatman, A. G., 2018, "Energy Transfer Mechanisms in the Ranque-Hilsch Vortex Tube," 2018 Canadian Society for Mechanical Engineering (CSME) International Congress, York University, Toronto, Ontario, Canada, May 27-30. 